\title{
Cartographie de dose par tissu aluminé. Essais en champ mixte $(\mathbf{n}, \gamma)$ ou $(\beta, \gamma)$
}

\author{
M. BENABDESSELAM*, P. IACCONI*, D. LAPRAZ* \\ A. SERBAT**, J. DHERMAIN**
}

(Manuscrit reçu le 22 avril 1997, révisé le 21 juin 1997, accepté le 12 septembre 1997)

RÉSUMÉ Lors d'une irradiation accidentelle due à des rayonnements ionisants, il est primordial d'avoir une estimation de la dose reçue par les personnes irradiées et de pouvoir reconstituer la cartographie de la dose absorbée. Compte tenu de l'extrême importance de ces données, un tissu de coton recouvert d'une alumine sensible aux photons $\gamma$ a été élaboré puis étudié en thermoluminescence.

ABSTRACT Cartography of absorbed doses by dosimetric textile. Testing in (n, $\gamma)$ or $(\beta, \gamma)$ mixed field.

When an accident due to ionising radiations occurs, it is very important to be able to map the surface cartography and to have an estimation of the dose absorbed by the irradiated persons. Since the importance of these informations, a coton textile coated with a dosemetric alumina has been settled and studied by thermoluminescence.

\section{Introduction}

Dans le but de reconstituer la cartographie de la dose absorbée ${ }^{(1)}$ lors d'une irradiation accidentelle, un tissu dosimétrique spécialement conçu pour l'habillement des personnes soumises aux risques d'irradiation, a été élaboré par le LPES-CRESA en collaboration avec l'IPSN et l'Institut textile de France (ITF) de Mulhouse. Ce tissu est constitué d'un support textile en coton sur lequel a été déposée, moyennant un liant et un épaississant, une couche d'alumine $\alpha$. Cet ensemble constitue ce que l'on appelle le «coton aluminé ».

Lorsqu'elle est soumise à une irradiation appropriée, l'alumine $\alpha$ incluse dans le coton emmagasine une énergie qu'elle restituera par chauffage sous forme de thermoluminescence (TL). L intensité du phénomène de TL étant fonction de la dose absorbée, le coton aluminé possède ainsi des qualités dosimétriques.

Un des objectifs fixés concerne l'amélioration des propriétés dosimétriques du coton aluminé telles que l'abaissement de son seuil de détection, son utilisation en

* LPES-CRESA, Université de Nice-Sophia Antipolis, Parc Valrose 06108, Nice Cedex 2, France

** DCE/CEB/DPN/DE, Le Bouchet, B.P. n ${ }^{\circ} 3,91170$ Vert-le-Petit, France

(1) Dose absorbée dans le tissus humain, donnée en terme d'énergie absorbée par unité de masse (Gy) 
champs mixtes (n, $\gamma)$ et $(\beta, \gamma)$, sa réponse de TL en fonction de l'énergie du rayonnement incident et enfin, sa tenue aux conditions climatiques, au lavage, aux frottements, etc.

Ce travail, effectué en collaboration avec le Centre d'Études du Bouchet (CEB), fait le point sur l'état des recherches actuelles sur la cartographie de dose par coton aluminé. Nous rappelons tout d'abord les objectifs que nous nous étions fixés, nous donnons ensuite quelques résultats de dosimétrie $\gamma$ en champs mixtes $(\mathrm{n}, \gamma)$ et $(\beta, \gamma)$ ainsi que la réponse de TL en énergie du coton aluminé.

L'utilisation en tant que vêtement dosimétrique, c'est-à-dire sous forme de blouse de travail, dont les premières mesures ont confirmé la faisabilité, requiert des propriétés de confort (souplesse, perméabilité à la sueur, etc.) comparables à celles des tissus commerciaux. Ceci entraîne que la charge en alumine doit rester inférieure à une valeur limite au delà de laquelle le tissu devient rêche et désagréable à porter. On peut contourner ce désagrément en utilisant le tissu sous forme de «patches » judicieusement disposés sous la doublure d'un vêtement. Le coton peut alors être chargé davantage en alumine.

\section{Conditions expérimentales}

La méthode expérimentale utilisée pour la lecture par thermoluminescence des cotons aluminés a déjà été décrite lors de précédentes communications (Lapraz et al., 1990 ; Benabdesselam et al., 1996). Les patches sont constitués par des morceaux de coton aluminé de $(4 \times 4) \mathrm{cm}^{2}$.

\section{Résultats et discussion}

\subsection{Dosimétrie $\gamma$ en champ mixte $(n, \gamma)$}

Dans le but de déterminer leur orientation par rapport à un faisceau de rayonnement mixte (n, $\gamma$ ), des mannequins anthropomorphes «PLASTINAUT » (matériau plastique équivalent tissu humain) sur lesquels ont été disposés des patches de coton aluminé ont été irradiés par le CEB dans diverses postures inconnues du LPES-CRESA dans le champ de rayonnement mixte du réacteur SILENE du centre CEA de Valduc (Tournier et al., 1994).

L'alumine étant insensible aux neutrons de fission du réacteur, la détermination par TL des doses $\gamma$ reçues par chacun de ces patches a permis de reconstituer la position des mannequins par rapport à la source et de déterminer la valeur de la dose $\gamma$ reçue aux divers points de mesures.

Parmi les diverses postures étudiées, nous donnons ici deux exemples. Sur chacun d'eux, le mannequin a été revêtu de 16 patches. 


\section{CARTOGRAPHIE DE DOSE PAR TISSU ALUMINÉ}
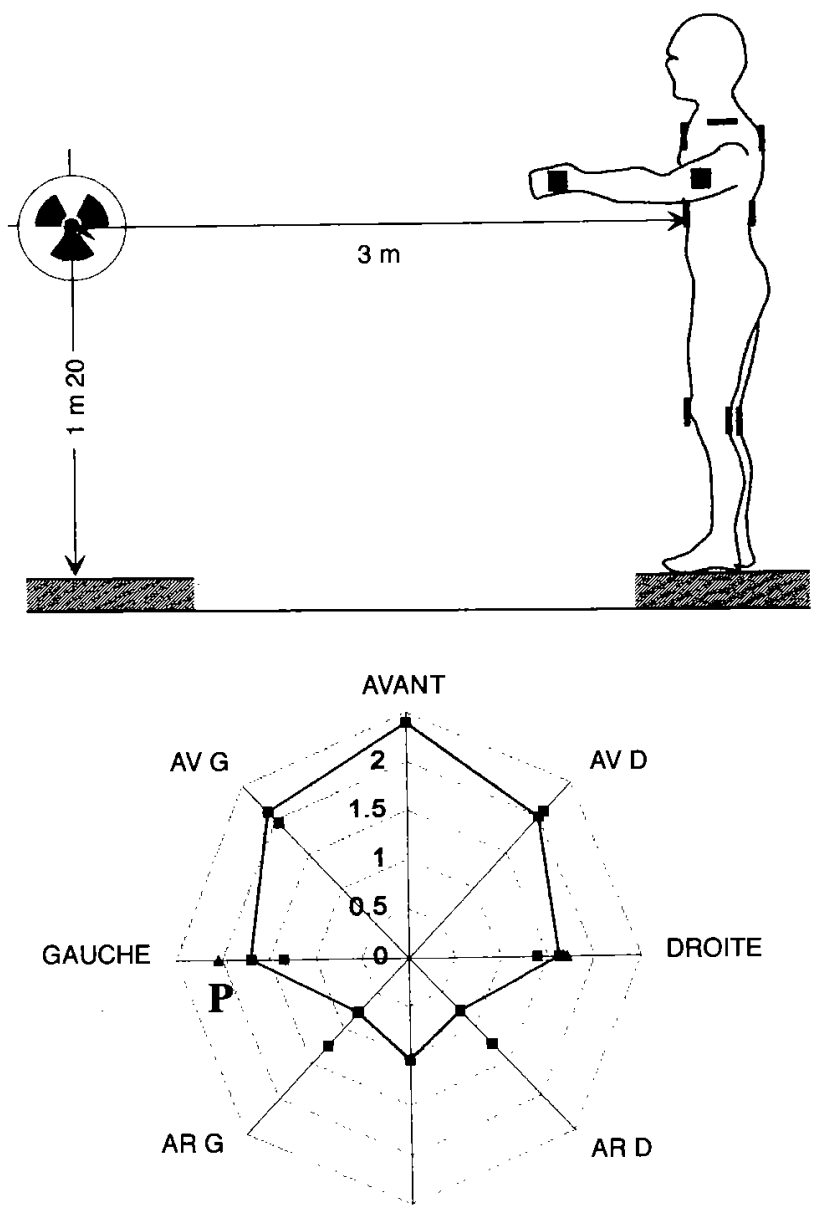

ARRIËRE

Fig. 1. - Le diagramme polaire montre que le mannequin a été irradié sur la face avant. P désigne le poignet gauche.

The polar diagram shows that the mannequin has been irradiated on the front side. P for the left wrist.

\subsubsection{Posture 1}

Les résultats obtenus par thermoluminescence ont donné lieu au diagramme polaire (Fig. 1) sur lequel sont représentées les valeurs des doses. Nous constatons que la dose la plus élevée se trouve sur l'avant de l'abdomen suivie respectivement par 
celles lues sur l'avant gauche puis sur l'avant droit du thorax. Viennent ensuite les doses lues sur le bras gauche puis sur le bras droit. Les doses à l'arrière et sur les côtés sont plus faibles. Le mannequin a donc été irradié face avant vers la source.

Nous remarquons de plus que les doses lues sur l'avant gauche du thorax et sur le bras gauche sont légèrement supérieures à celles lues sur les parties homologues droites, et que la dose lue sur le poignet gauche est plus importante que celles lues sur l'épaule gauche ou le bras gauche.

Nous en avons conclu que le mannequin avait son bras gauche tendu à l'horizontale face à la source. La confirmation de cette posture nous a été communiquée par le CEB à la fin des mesures.

\subsubsection{Posture 2}

Sur ce deuxième exemple, le diagramme polaire (Fig. 2) montre une dose reçue plus importante sur le côté droit que sur les autres parties du mannequin. On peut donc dire qu'il a été irradié par le profil droit. De plus, sur le côté gauche, la dose reçue par le poignet est plus élevée que celles reçues par les autres parties. Ceci nous conduit à supposer que le mannequin avait son profil droit face à la source et que son bras gauche était tendu à l'horizontale. Là aussi confirmation nous a été donnée par la suite.

\subsection{Diminution du seuil de détection}

Dans le contexte initial du développement de la blouse de travail, le tissu aluminé devait, tel un tissu normal, satisfaire au critère de confort. Une des façons de respecter ce critère était d'appliquer un faible dépôt en alumine, de manière à conserver toute sa souplesse au tissu. La conséquence était que, pour un tissu chargé à $25 \mathrm{~g} \mathrm{~m}^{-2}$, le seuil de détection était de $0,25 \mathrm{~Gy}$, ce qui nous paraissait élevé. Dans la mesure où on décide d'utiliser le coton aluminé sous forme de patches, on peut diminuer le seuil de détection en augmentant la teneur en alumine. Avec des cotons dont la teneur est de $65 \mathrm{~g} \mathrm{~m}^{-2} \mathrm{~d}$ 'alumine, nous avons pu facilement détecter des doses $\gamma$ de l'ordre de $0,1 \mathrm{~Gy}$.

Pour illustrer l'effet du dépôt en alumine, nous avons représenté (Fig. 3) la variation de l'intensité de TL observée sur 7 échantillons de cotons aluminés de teneur en alumine variant de 0 à $65 \mathrm{~g} \mathrm{~m}^{-2}$ et irradiés par un faisceau de rayons $X$. Les rayons $X$ utilisés sont issus d'un tube à anticathode de tungstène, alimenté sous $45 \mathrm{kV}$ et filtré par un écran d'aluminium de $0,5 \mathrm{~mm}$ d'épaisseur. Le produit étudié était déposé sur une platine métallique d'irradiation et le kerma dans l'air maintenu constant et égal à $3 \mathrm{~Gy}$. Nous pouvons constater qu'entre les cotons de 25 et $65 \mathrm{~g} \mathrm{~m}^{-2}$ en alumine, la réponse du détecteur de TL est multipliée par un facteur 3 (Benabdesselam et al., 1996). 

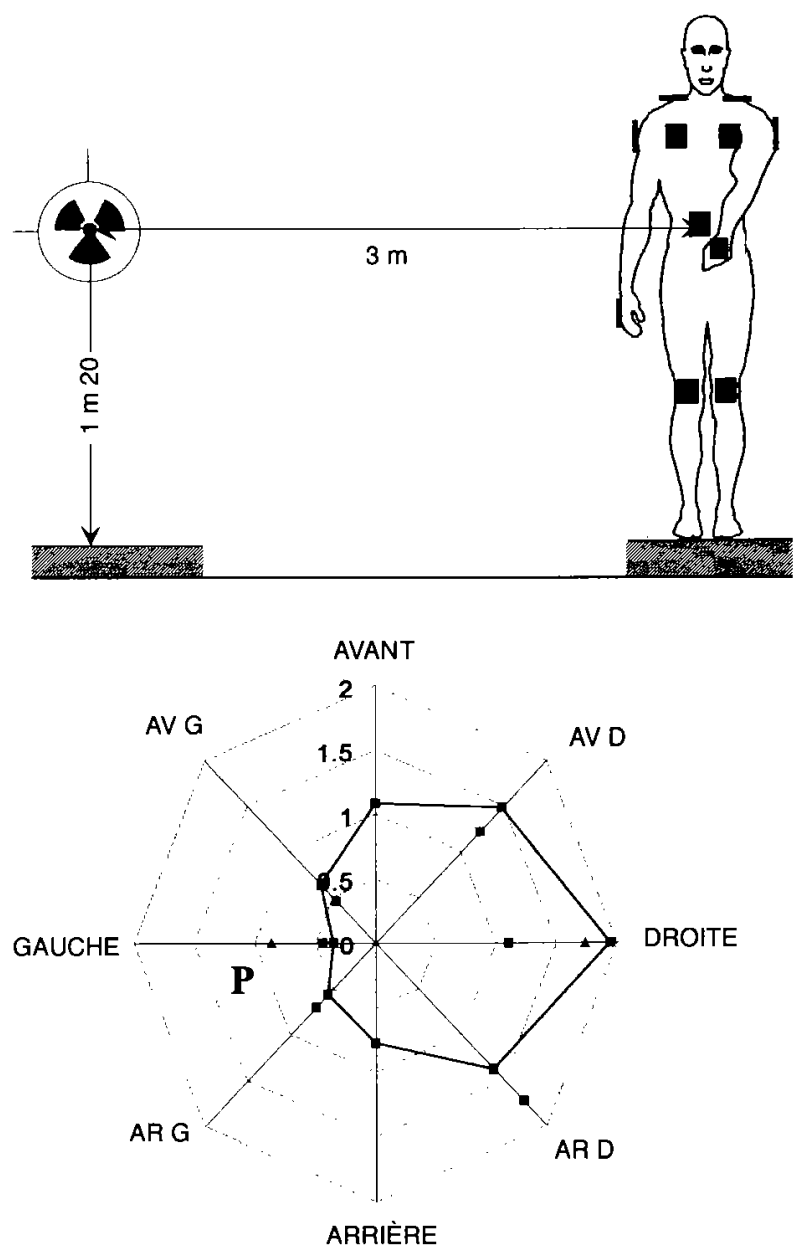

Fig. 2. - Le diagramme polaire montre que le mannequin a été irradié sur le profil droit. P désigne le poignet gauche.

The polar diagram shows that the mannequin has been irradiated on the right side. P for the left wrist. 
M. BENABDESSELAM et al.

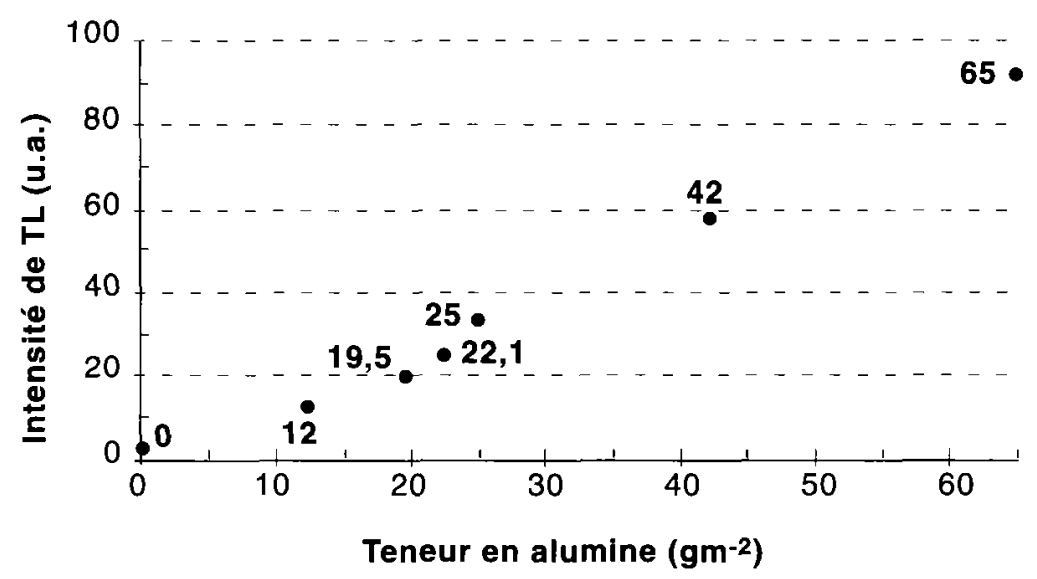

Fig. 3. - Variation de l'intensité de thermoluminescence en fonction de la teneur en alumine. Variation of thermoluminescence intensity as a function of alumina content.

\subsection{Influence de la lumière ambiante et de la température}

Les mesures d'affaiblissement (fading) sur le pic dosimétrique du coton chargé à $65 \mathrm{~g} \mathrm{~m}^{-2}$ après irradiation par des rayons $\mathrm{X}$ avec un kerma dans l'air de 5,9 Gy, ont donné lieu aux résultats représentés sur la figure 4 qui représente le rapport des lectures à l'instant $t$ aux lectures à l'instant $t=0$.

- un déclin de $10 \%$ est observé en thermoluminescence sur une durée de stockage de deux mois à la température ambiante et dans l'obscurité totale (Fig. 4a).

- si le stockage est fait à température ambiante sous exposition d'une lampe à incandescence de $40 \mathrm{~W}$ située à 1 mètre, la perte d'information est alors de $18 \%$ sur une durée approximativement identique (Fig. $4 \mathrm{~b}$ ).

\subsection{Réponse en énergie aux photons}

Le coton chargé à $65 \mathrm{~g} \mathrm{~m}^{-2}$ a été irradié par des photons d'énergie variant entre 45 et $204 \mathrm{keV}$ et par ${ }^{137} \mathrm{Cs}$ et ${ }^{60} \mathrm{Co}$ à kerma dans l'air constant. Pour chaque énergie, sa réponse relative à la réponse obtenue au ${ }^{60} \mathrm{Co}$ a été calculée. Elle est intermédiaire entre celles de l'alumine pure (Portal, 1978) et de l'alumine dopée au carbone TLD 500 (Fig. 5).

Pour interpréter ce résultat, nous envisageons de réaliser une simulation par la méthode EGS4 afin de prendre en compte le rôle joué par les différents constituants du coton aluminé dans l'absorption des photons $\gamma$. 


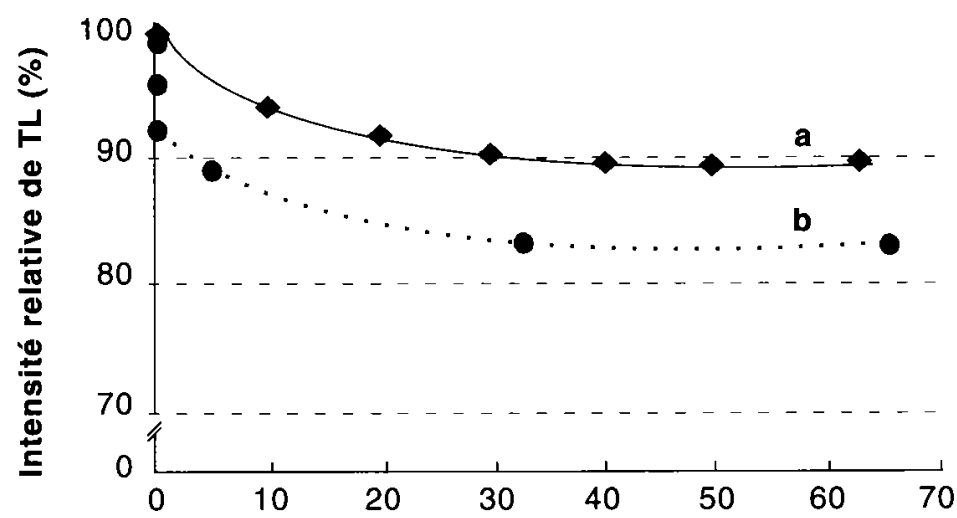

Durée $t$ (jours)

Fig. 4. - Affaiblissements thermique et optique, en fonction :

a) de la durée du stockage à l'obscurité et à la température ambiante

b) de la durée d'exposition à une lampe à incandescence de $40 \mathrm{~W}$.

Thermal and optical fadings, versus:

a) the storage time in the dark and at room temperature

b) the time of exposure to $40 \mathrm{~W}$ incandescent lamp.

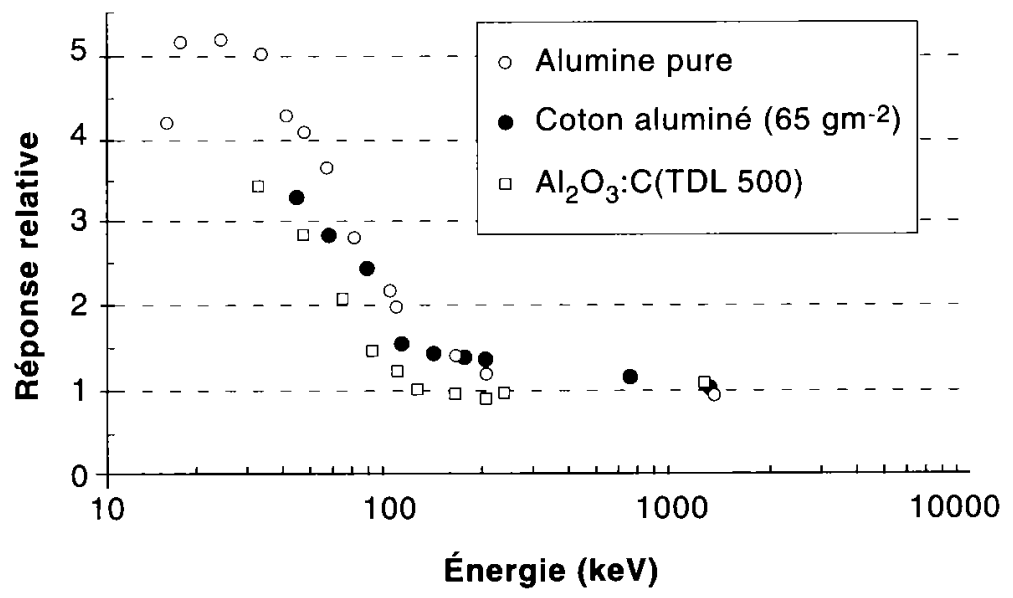

Fig. 5. - Réponse relative au ${ }^{60}$ Co de l'intensité de thermoluminescence du coton aluminé dont la teneur en alumine est de $65 \mathrm{~g} / \mathrm{m}^{2}$.

Relative response of thermoluminescence intensity to ${ }^{60} \mathrm{Co}$ of the $65 \mathrm{~g} / \mathrm{m}^{2}$ coated dosimetric cotton. 

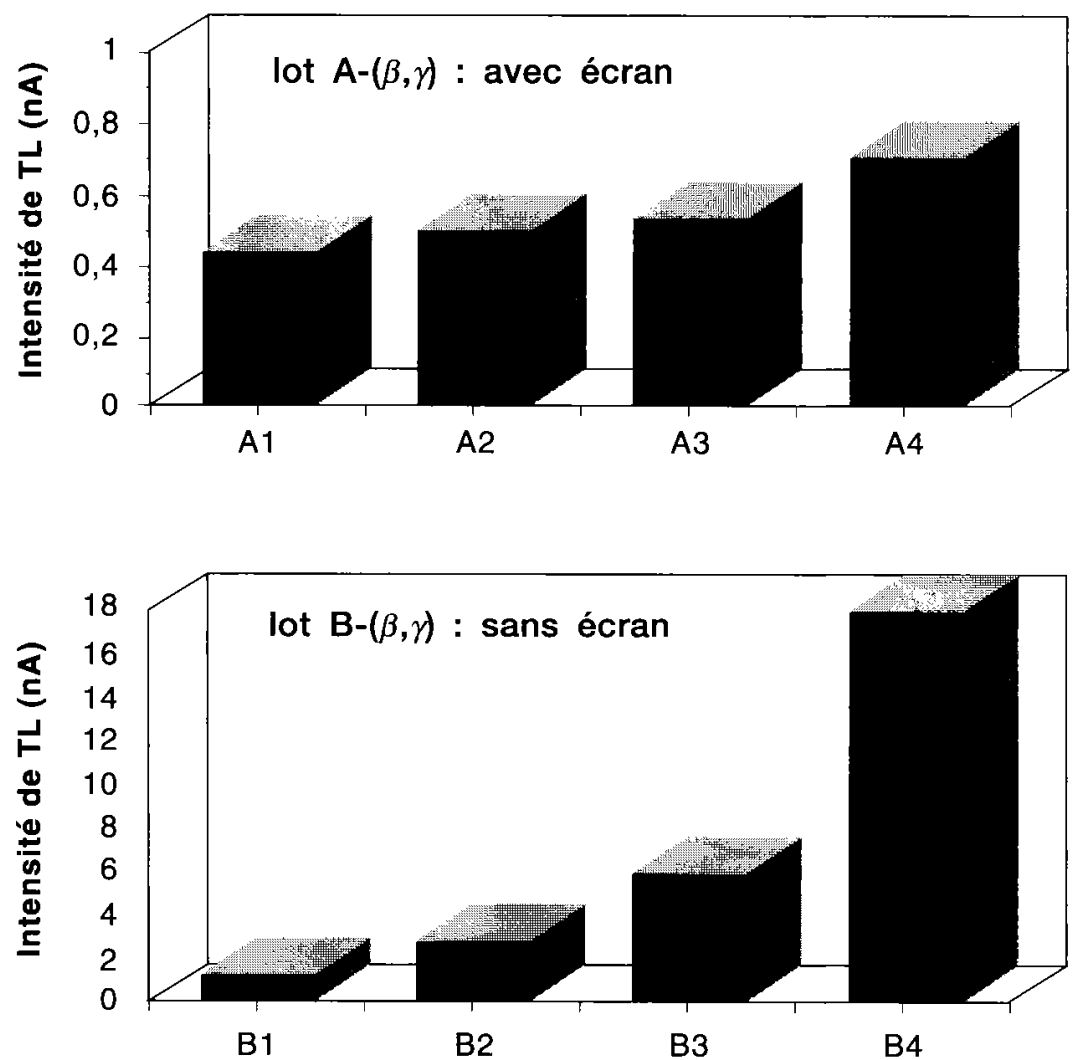

Fig. 6. - Réponse en intensité de thermoluminescence de quatre échantillons de coton aluminé en présence (lot $A$ ) et en absence (lot B) d'un écran de plexiglas de $10 \mathrm{~mm} d$ 'épaisseur. Thermoluminescence intensity reponse of four dosimetric cotton pieces with (lot $A)$ and without (lot B) a $10 \mathrm{~mm}$ thick plexiglass shield.

\subsection{Dosimétrie en champ mixte $(\beta, \gamma)$}

Deux lots ( $\mathrm{A}$ et $\mathrm{B}$ ) de coton répartis chacun en quatre échantillons numérotés de 1 à 4 ont été utilisés. Quatre paires d'échantillons ont été disposées à 4 distances différentes de la source et irradiées devant une source de ${ }^{140} \mathrm{La}$ qui émet un rayonnement $\beta\left(E_{\min }=1,2 \mathrm{MeV}, E_{\max }=2,16 \mathrm{MeV}\right)$ et un rayonnement $\gamma$ $\left(E_{\min }=0,33 \mathrm{MeV}, E_{\max }=2,5 \mathrm{MeV}\right)$. Pour chacune des paires d'échantillons, l'un était « nu », l'autre coiffé d'une lame de plexiglas qui arrête le rayonnement $\beta$. Les résultats reportés sur la figure 6 montrent un rapport d'intensité de thermoluminescence de l'ordre de 20 entre les échantillons B4 et A4.

Il s'agit là de résultats préliminaires. 


\section{Conclusion}

L'objectif concernant l'abaissement du seuil de détection à $0,1 \mathrm{~Gy}$ est atteint, grâce à l'utilisation de cotons aluminés dont la teneur en alumine est de $65 \mathrm{~g} \mathrm{~m}^{-2}$. Trop rugueux pour être porté à même la peau, ce produit doit être utilisé sous forme de patches insérés sous la doublure d'un vêtement, par exemple.

Notons que dans le cas d'un accident de criticité, il n'est cependant pas nécessaire d'avoir un seuil de détection aussi bas que 0,1 Gy et que d'autres méthodes d'amélioration du seuil sont également possibles telles que, par exemple, l'augmentation de la surface vue par le détecteur de TL.

\section{RÉFÉRENCES}

Benabdesselam M., Iacconi P., Lapraz D., Serbat A., Laugier J., Dhermain J. (1996) Qualification of Specially Prepared Clothing Material for Gamma Dosimetry: Application to Mixed NeutronPhoton Radiation Fields, Radiat. Prot. Dosim., 66, 125-128.

Lapraz D., Iacconi P., Alessandri M.F., Barthe J., Portal G. (1990) Dosimetric Characteristics of Specially Prepared Clothing Material, Radiat. Prot. Dosim., 33, 131-134.

Portal G. (1978) Étude et Développement de la Dosimétrie par Radiothermoluminescence. Thèse, Université de Toulouse.

Tournier B., Barbry F., Rozain J.P. (1994) SILENE, a New Radiation Reference Source. Topical Meeting on Physics, Safety and Applications of Pulse Reactors, Washington, DC, 72-79. 